\title{
Surface texturing of dental implant surfaces with an ultrafast fiber laser
}

\author{
B. Oktem ${ }^{1}$, H. Kalaycioglu ${ }^{2}$, M. Erdoğan ${ }^{1}$, S. Yavaș ${ }^{1}$, P. Mukhopadhyay ${ }^{2}$, U. H. Tazebay ${ }^{3}$, Y. Aykaç ${ }^{4}$, \\ K. Eken ${ }^{5}$, F. Ö. Ilday ${ }^{2}$ \\ ${ }^{1}$ Materials Science and Nanotechnology Graduate Program, Bilkent Universty, 06800 Ankara, Turkey \\ ${ }^{2}$ Department of Physics, Bilkent Universty, 06800 Ankara, Turkey \\ ${ }^{3}$ Molecular Biology and Genetics Department, Bilkent University, 06800 Ankara, Turkey \\ ${ }^{4}$ Faculty of Dentistry, Ankara University, 06500 Ankara, Turkey \\ ${ }^{5}$ FiberLAST, Ltd., 06531 Ankara, Turkey
}

\begin{abstract}
Controlled modification of implant surfaces using femtosecond, picosecond and nanosecond pulses from home-built all-fiber-integrated lasers is demonstrated. Picosecond and femtosecond pulses offer superior control over the surface texture. Increasing cell attachment to surface is discussed.

(C)2010 Optical Society of America
\end{abstract}

OCIS codes: (140.3390) Laser materials processing; (140.3510) Lasers, fiber; (170.1850) Dentistry.

Laser texturing of material surfaces is increasingly used to improve tribological and biomechanical properties of surfaces since it is fast, harmless to the environment and offers excellent precision [1]. For example, texturing of implant surfaces is used to increase cell attachment and subsequent proliferation, since surface roughness plays an important role in cell adhesion to surfaces of orthopedic prostheses and dental implants [2]. However, the techniques commonly used in commercial applications are mechanical methods (machining and sandblasting) and chemical methods (acid etching, oxidation), all of which have undesirable contamination and environmental issues and lack the precision of laser surface modification. The thermal effects of long-pulsed and CW lasers are undesirable in certain applications and the prohibitively high-cost and complexity of ultrashort pulsed lasers have confined their application to surface texturing to laboratory experiments [3].

Ultrafast fiber systems are emerging as lower-cost, more robust alternatives to their solid-state counterparts. However, there are relatively few reports on material processing and none on surface texturing with ultrafast fiber lasers, to our knowledge. Here, we demonstrate the use of all-fiber-integrated fiber amplifier systems, developed in our laboratory using standard components, for precise and highly repeatable implant surface texturing using picosecond and femtosecond pulses. Various surface textures with micron and nanometer size features can be created with high repeatability. For comparison, a nanosecond fiber laser is also used, which exhibits increased thermal effects, as expected.

The experimental setup is illustrated in Fig. 1. Two fiber laser-seeded ultrafast all-fiber-integrated amplifier systems delivering picosecond or femtosecond pulses, respectively, are used. The details of these systems are described in [4] and [5]. Apart from a small difference in their central wavelengths (1030 nm vs. $1060 \mathrm{~nm}$ ), repetition rates, and pulse durations, the two lasers are quite similar in architecture. The picosecond fiber laser produces $\sim 20$ ps-long pulses with more than $10 \mathrm{~W}$ of average power at $40 \mathrm{MHz}$ repetition rate. The femtosecond fiber laser delivers 140 fs-long, $1 \mu \mathrm{J}$-energy pulses at $1 \mathrm{MHz}$ repetition rate. The laser beam is focused to a spot size of $\sim 15 \mu \mathrm{m}$ and the samples are placed on a computer-controlled 3D motorized stage. For comparison, a nanosecond fiber laser (FL-NS-10W, FiberLAST) producing $70 \mathrm{~ns}-$ long pulses at $25 \mathrm{kHz}$ repetition rate and maximum average power of $8 \mathrm{~W}$ is used with a galvanometer-based scanner to form the patterns.

A large variety of surface patterns can be created using both the picosecond and femtosecond pulses. With picosecond pulses, microstructures with features sizes of 10-20 $\mu \mathrm{m}$ and height differences of $\sim 5 \mu \mathrm{m}$ are easily created (Fig. 2). Raman spectroscopy analysis reveals that titanium is transformed into titanium oxide under the effect of the laser. Using femtosecond pulses, in addition to similar microstructures, surface roughness in the 50$100 \mathrm{~nm}$ range can be created, if the focused intensity is kept sufficiently low (Fig. 3). This was not found to be possible with picosecond pulses, which we attribute to the greatly reduced, but enduring thermal effects with picosecond pulses. There is no discernible heat affected zone (HAZ) when using femtosecond pulses. For most surface texturing applications, e.g., creation of microdimples, the micron-scale control afforded by the picosecond fiber laser is sufficient [6]. However, comparison with nanosecond duration pulses reveals a markedly different process, with a more pronounced HAZ (Fig. 4).

The effect of the laser surface texturing on cell attachment was tested with cell-culture experiments. We compare the laser-textured surfaces with three different commercially obtained implant surface types, textured using sand-blasting, acid-etching and the SLA method. In these experiments, Saos-2 cells are cultured and then seeded onto 8-mm Ti disk surfaces. After 36 hours of incubation, a post-treatment process is applied to eliminate cells, which are not attached strongly to the surface. The cell numbers are then counted under a fluorescence microscope for each disk. The results have large statistical deviations, but it can be seen that the cell adhesion on 


\section{JTuD15.pdf}

all of these surfaces is comparable within statistical fluctuations. In addition, we observe that the cells tend to align with any linear microfeatures on the laser-textured surface, a phenomenon known as contact guidance.
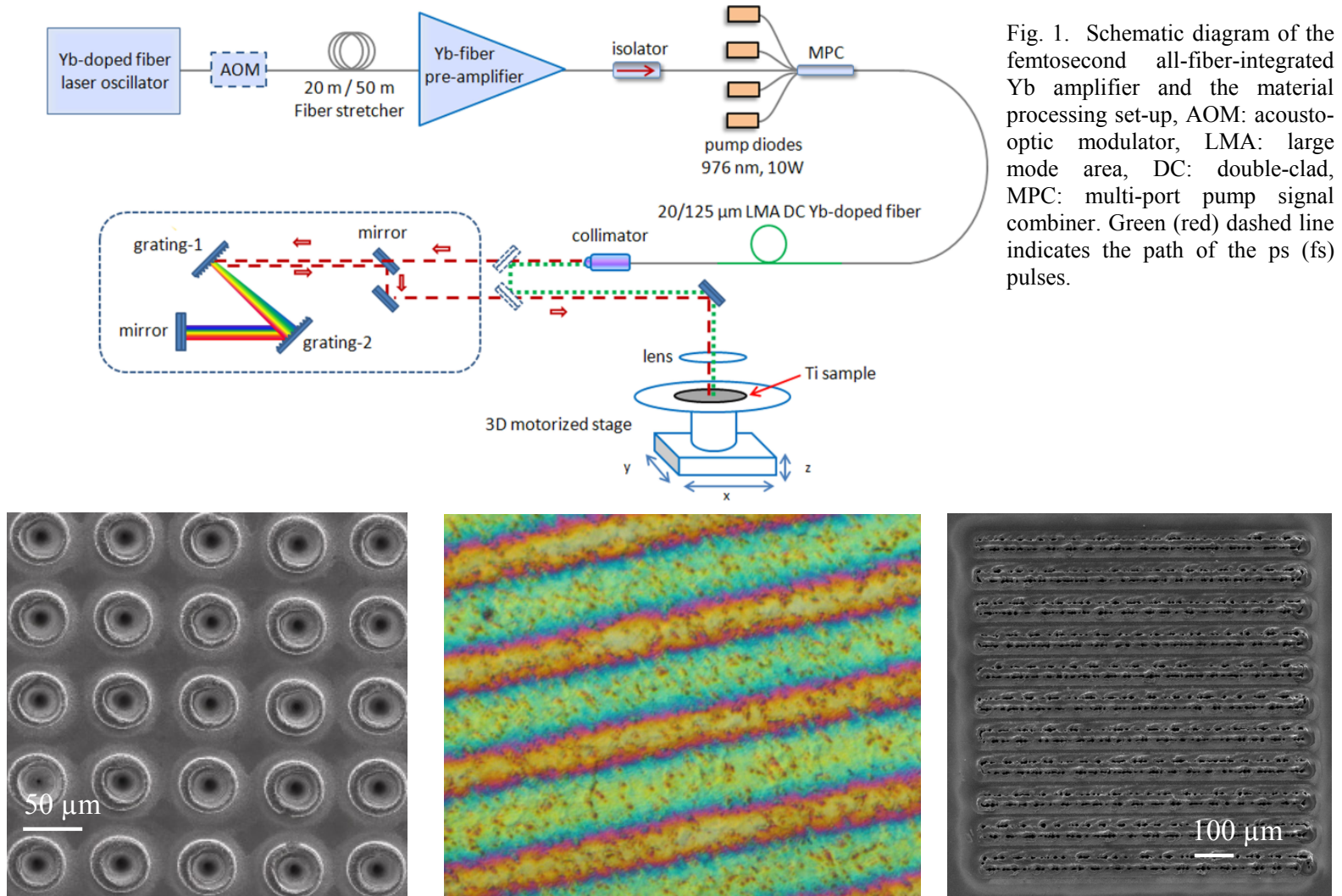

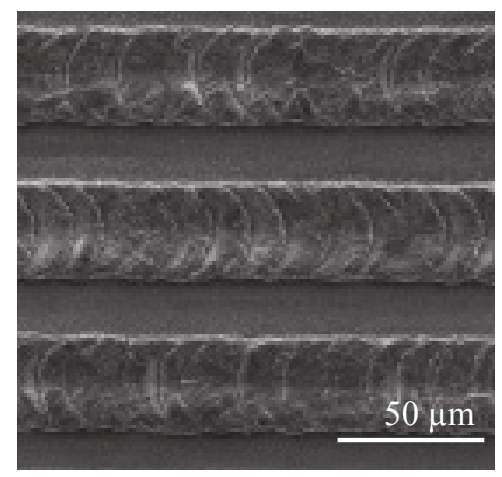

Fig. 2. Scanning electron microscope images of the micron-scale surface textures created using the picosecond fiber laser.

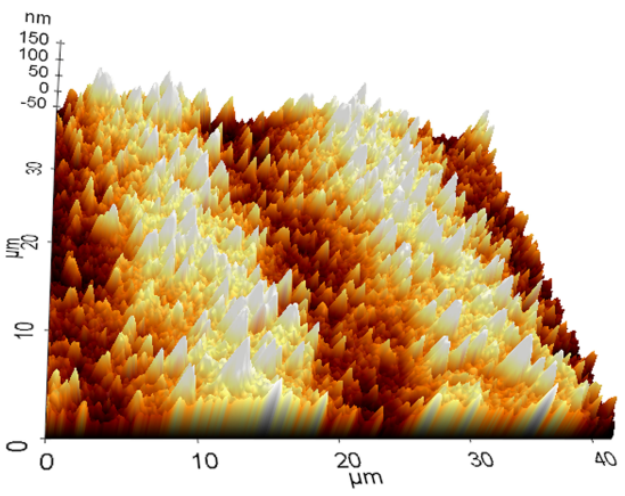

Fig. 3. Optical (top) and atomic force (bottom) microscope images of the nanometer-scale surface textures created using the femtosecond fiber laser.

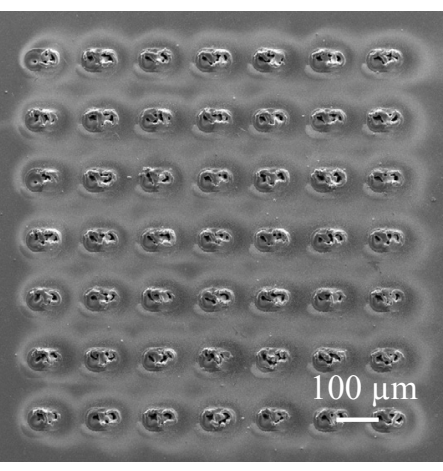

Fig. 4. Scanning electron microscope images of linear (top) and dotted (bottom) patterns formed with nanosecond pulses.

In conclusion, we have demonstrated controlled surface texturing of Ti dental implant surfaces using fiber lasers delivering picosecond and femtosecond pulses. Comparison of the surface textures made with a nanosecond laser demonstrates the advantages of ultrashort pulses through minimizing thermal effects and increased precision. Initial cell attachment experiments demonstrate performance comparable to commercially prepared surfaces with non-laser-based techniques. Significantly, for micron-scale surface texturing, we find that the picosecond pulses perform virtually the same as femtosecond pulses. Given that the former does not require a grating compressor, and the laser beam is directly delivered from the optical fiber, use of picoseconds pulses at $\mathrm{MHz}$ repetition rates holds great potential, particularly for in vivo applications. While our emphasis has been on texturing of Ti-based dental implant surfaces, these results can easily be adapted to different materials and textures.

[1] I. Etsion, "State of the art in laser surface texturing," J. Tribol. 127, 248 (2005).

[2] L. Hao, J. Lawrence, Y. F. Phua, K. S. Chian, G. C. Lim, H. Y. Zheng, "Enhanced human osteoblast cell adhesion and proliferation on 316 LS stainless steel by means of CO2 laser surface treatment," J. Biomed. Mater. Res. B Appl. Biomater. 73B, 146-156 (2005).

[3] A. Y. Vorobyev and C. Guo, "Femtosecond laser nanostructuring of metals," Opt. Express 14, 2164 (2006).

[4] P. K. Mukhopadhyay, K. Özgören, I. L. Budunoglu, and F. Ö. Ilday, IEEE J. Sel. Top.Quant. 15, 145 (2009).

[5] H. Kalaycioglu, B. Oktem, C. Senel, P. P. Paltani, F. Ö. Ilday, "Micro joule-energy, 1 MHz-repetition rate pulses from an all-fiberintegrated nonlinear chirped-pulse amplifier," under review at Optics Letters.

[6] I. Etsion, "Improving tribological performance of mechanical components by laser surface texturing," Tribol. Lett. 17, 733 (2004). 